\title{
Drug Resistance Associated with Blood Borne Bacteria in Dhaka Metropolis
}

\author{
Tasnia Ahmed ${ }^{1}$, Rajib Dey ${ }^{1}$, Rashed Noor ${ }^{2 *}$ \\ ${ }^{1}$ Department of Microbiology, Stamford University Bangladesh, 51 Siddeswari Road, Dhaka 1217, Bangladesh, ${ }^{2}$ Independent University Bangladesh. School of \\ Life Sciences (SLS). Plot 16, Block B, Aftabuddin Ahmed Road, Bashundhara, Dhaka-1229
}

\begin{abstract}
Blood borne infections are easy to contract during hospital procedures and can easily be transferred to other personnel in a healthcare setting. Bacteremia has become a common incidence and treatment is by administration of antibiotics. But this is not a simple task any more, since the pathogenic bacteria causing bacteremia are becoming resistant to a wide range of antibiotics. Blood samples were collected from outdoor patients seeking laboratory tests in Dhaka city. Blood samples were inoculated onto blood agar medium and after incubation for $37^{\circ} \mathrm{C}$ for 24 hours, bacterial isolates were identified and subjected to antibiotic susceptibility test by following the Kirby-Bauer method. The current present study deals with 100 patients of bacteremia from whom 150 isolates of 15 different bacterial genera have been collected among which the most prominent bacteria were Staphylococcus spp. (51 isolates), Pseudomonas spp. (19 isolates) and Escherichia spp. (19 isolates). After the antibiotic susceptibility test it was found that all isolates were resistant to a number of commonly used antibiotics. Twenty eight different antibiotics were used for this study. All isolates showed resistance to CAR, ATM, TOB, CXM, FD, CL, CAZ, AMC. NET and CN showed the most effective results (can effectively clear 9 and 10 types of isolates respectively found in this study). New discovery of drugs to fight these resistant pathogens is needed. In the meantime, safe administration of drugs, handling patients with appropriate protective personal clothing and apparatus, proper waste disposal managements in the hospital should be maintained strictly.
\end{abstract}

Key words: Antibiotic, pathogen, drug resistance, bacteremia.

\section{Introduction}

Systemic infections with the involvement of blood contaminated with pathogenic microorganisms is a serious issue which often leads to difficulty in treatment as well as high mortality rate ${ }^{1,2}$. Microorganisms can gain entrance through different ways. A good number of microorganisms from both gram positive and gram negative groups has been confirmed to cause bacteremia- bacterial infection in blood. Staphylococcus aureus, Streptococcus pneumoniae, Streptococcus anginosus, Salmonella spp., Pseudomonas aeruginosa, Acinetobacter spp, Enterobacter spp., Corynebacterium spp, Bacillus spp, Clostridium spp., Haemophilus influenzae, Neisseria meningitidis, Rhodococcus equi., Streptococcus bovis, Escherichia coli, Aeromonas spp., Serratia marcescens, Listeria monocytogenes ${ }^{3-6}$. Treatment of bacteremia is quite difficult specially in case of gram positive bacteria ${ }^{5}$. Among them MRSA- methicillin resistant Staphylococcus aureus is the most common causing bacteremia in European countries capable of causing infective endocarditis and metastatic infections ${ }^{7-10}$. The factors responsible for transportation of the bacteria includes central venous catheter, urinary catheter, cellulitis, ulcer, injecting drugs, surgical wounds, immunosuppression etc ${ }^{11-13}$. The other most prominent community acquired as well as common nosocomial bacteremia in United States is Escherichia coli ${ }^{14-19,5}$. Though E. coli is a commensal in human body, under some circumstances it causes opportunistic infection. Predisposing factors include people over 65 years old, specially women. The portal of entry was mostly urinary tract and gastrointestinal tract ${ }^{20}$.

Bacteremia occurs mainly in patients with immune deficiencies. One major cause of such immunodeficiency is HIV infection. Some factors influence the bacteremic condition such as central venous catheters, high rate of intravenous drug administration, defects in cell mediated immunity (CD-4), neutropenia etc. Some portals of entry includes genitourinary tract, respiratory tract, gastrointestinal tract, broken skin etc. A common problem is due to non-typhoidal Salmonella $\mathrm{spp}^{21-22}$. Though the morbidity and mortality rate is high two steps can be taken at a time to lessen the situation. One involves antiretroviral drug administration for controlling HIV and adequate prolonged antibiotic treatment for the bacteremia ${ }^{23-26}$.

Piercing and tattoo involves with the injections which come in contact with blood. In case of tattoo colors are injected under the skin and there is a great chance of contamination during the whole process $^{27-30}$. The needle used for tattoo once used for a person infected with $\mathrm{HBV}, \mathrm{HCV}$, HIV etc can transmit to other person while injecting $31-37$.

*Corresponding author:

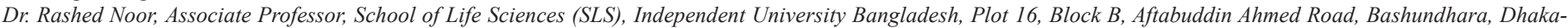
1229, Cell: +8801749401451,E-mail:noorrashed@yahoo.com 
Health care workers are at a high risk of exposure to blood borne pathogenic microorganisms from the infected patient during some medical procedures involving sharp needles, scissors, or any broken sharps contaminated with blood of the patient. After that, the health care workers further disseminate the infection to other patients through the same process. About $25 \%$ cases bacteremia are hospital acquired ${ }^{38}$.

Pathogenesis of bacteremia occurs step by step. For example, Staphylococcal isolates at first adhere and colonize through broken skin and mucus membrane following connection with blood. Adherence factor is also a virulence factor for this bacteria. They produce different enzymes and toxins to hydrolyze tissue and disseminate towards other parts through blood. Such protein material are recognizable as virulence factors ${ }^{39-43}$.

To diagnose the bacteremic condition some tests should be done to detect the pathogen. The diagnostic tests include transthoracictransesophageal echocardiography, radiography, positronemission tomography, CT, WBC count, C-reactive protein level measurement etc to detect deep seated infections. The samples are blood samples collected from venous catheters. Blood cultures to identify pathogens followed by PCR and fluorescent based methods are popular for diagnostic purposes. The infection responsible to cause bacteremia should be identified. Sometimes local pus, abscess, scars etc physical findings occurs after the systemic condition which makes the phenomena even worst ${ }^{1,44-46}$.

The treatment starts right after the detection of positive test results. But if contamination occurs in the blood culture and shows a false positive result which actually is of no relation with the patient 47-49. In the same way, false negative test result is not desirable. Patients with blood infections may not show positive result increasing the mortality and morbidity rate because of proper treatments. This happens specially for pneumococcal infections. Presence of Pneumococci can be showed in blood culture within 12 hours but not after 24 hours. In case of counter immune electrophoretic method, the same phenomena happens; negative result after 24 hours. Streptococcus pneumoniae often shows such false negative result ${ }^{50}$.

In the current study about 100 patients were selected to determine bacterial isolates from both male and female patients of different ages and to determine drug sensitivity patterns of the isolates.

\section{Materials and method}

\section{Study area and sampling}

About 100 male and female patients were randomly selected who were of different ages. Patients were selected from Dhaka city seeking for treatments. The experiment was conducted within February, 2016 and May, 2016. Blood samples were collected aseptically and immediately transferred to different culture media. Personal protective equipment and clothing was strictly maintained to avoid any hazards and transmissions so healthcare workers and to others.

\section{Identification of bacterial isolates}

Bacterial isolates found in the blood agar plates were detected following the biochemical identification. Different biochemical tests like TSI, MIU, catalase, oxidase, citrate utilization test, MR, VP were conducted to confirm the identification of the isolated bacteria.

\section{Detection of antibacterial susceptibility pattern}

Blood samples were subjected to inoculate aseptically on nutrient agar and blood agar plates to find the bacterial colonies present in the blood sample. After 48 hours of incubation all the bacterial colonies were subjected to biochemical identification to get the identification of the blood borne bacteria. The main part of the case study was the susceptibility pattern of the isolates against the popular antimicrobials used in Bangladesh. For this work, bacterial suspensions of the isolated bacteria (Staphylococcus spp., Pseudomonas spp., Escherichia spp., Klebsiella spp., Proteus vulgaris, Acinetobacter spp., Citrobacter spp., Enterobacter spp., Enterococcus spp., Streptococcus spp., beta hemolytic Streptococcus spp., Moraxella spp. and Providentia spp.) were prepared in normal saline separately and incubated at $37^{\circ} \mathrm{C}$. After matching the suspensions with $0.5 \mathrm{McF}$ arland standard, they were inoculated onto the Mueller Hinton agar plates to make a lawn of bacterial suspension. After that 28 different antibiotic discs (Netilmicin, Trimethoprim-Sulphamethoxazole, Fusidic acid, Ciprofloxacin, Oxacillin, Vancomycin, Tobramycin, Carbenicillin, Imipenem, Cefalonium, Amoxicillin, Azithromycin, Ceftriaxone, Tigecycline, Cefotaxime, Cefoxitin, Cefuroxime, Piperacillin-Tazobactam, Ceftazidime, Cefepime, Fusidic acid, Clindamycin, Chloremphenicol, Amikacin, Colistin, Gentamicin, Rifampin, Radicicol) were aseptically placed over the bacterial lawn. After 24 hours incubation at $37^{\circ} \mathrm{C}$ plates were observed for the presence of the zone of inhibition and measured in millimeter scale to determine whether the pathogenic isolates were antibiotic resistant or sensitive.

\section{Results}

Isolated bacteria were subjected to biochemical test for proper identification after doing the tests we found Escherichia spp., Staphylococcus spp., Streptococcus spp., Pseudomonas spp., Klebsiella spp., Proteus spp., Providentia spp., Moraxella spp., Enterobacter spp., Citrobacter spp., Acinetobacter spp. and Enterococcus spp.

In the current study, 129 isolates of 12 types of bacteria collected from blood were subjected to antimicrobial susceptibility test to determine the drug resistance patterns. Most predominant isolate was Staphylococcus spp. (51 patients). Pseudomonas spp. (19 patients), Escherichia spp. (19 patients), Proteus spp. (12 patients) were also found in the bacteremic patients. The less frequent bacterial isolates found from the patients were Klebsiella spp., Acinetobacter spp., Citrobacter spp., Enterobacter spp., Enterococcus spp., Streptococcus spp., Moraxella spp. and Providentia spp. 
Table 1. Biochemical identification of the isolated bacteria.

\begin{tabular}{|c|c|c|c|c|c|c|c|c|c|c|}
\hline & $\frac{\vec{\Xi}}{\frac{\vec{G}}{\omega s}}$ & 茍 & ర్ల & 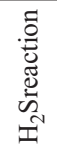 & $\begin{array}{l}\overrightarrow{0} \\
\frac{0}{0} \\
\frac{0}{0} \\
\frac{0}{\Xi}\end{array}$ & 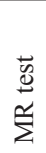 & $\begin{array}{c}\vec{w} \\
\stackrel{s}{s}\end{array}$ & 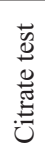 & 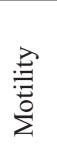 & $\begin{array}{l}\vec{w} \\
\stackrel{0}{0} \\
0 \\
0 \\
\frac{\tilde{g}}{\Delta} \\
0 \\
0\end{array}$ \\
\hline Klebsiella spp.Y & $\mathrm{Y}$ & - & - & - & - & - & - & - & + & \\
\hline Enterococcus spp. & $\mathrm{Y}$ & $\mathrm{Y}$ & - & - & - & - & + & - & - & - \\
\hline Staphylococcus spp. & $\mathrm{Y}$ & $\mathrm{R}$ & + & + & - & + & - & + & + & - \\
\hline Streptococcus spp. & $\mathrm{Y}$ & $\mathrm{Y}$ & - & - & - & + & - & - & - & - \\
\hline Citrobacter spp. & $\mathrm{Y}$ & $\mathrm{Y}$ & + & + & - & + & - & + & + & - \\
\hline Acinetobacter spp. & $\mathrm{R}$ & $\mathrm{R}$ & - & - & - & - & - & + & - & - \\
\hline Enterobacter spp. & $\mathrm{Y}$ & $\mathrm{Y}$ & + & - & - & - & + & + & - & - \\
\hline Moraxella spp.R & $\mathrm{R}$ & - & - & - & + & - & - & & + & \\
\hline Providentia spp. & $\mathrm{R}$ & $\mathrm{R}$ & & - & + & + & - & + & + & - \\
\hline Pseudomonas spp. & $\mathrm{Y}$ & $\mathrm{Y}$ & - & - & - & - & - & + & - & - \\
\hline Escherichia spp. & $\mathrm{Y}$ & $\mathrm{Y}$ & + & - & + & + & - & & & \\
\hline Proteus spp. Y & $\mathrm{Y}$ & + & + & - & - & + & + & + & + & \\
\hline
\end{tabular}

Table 2. Drug resistance trait of the isolates collected from blood sample.

\begin{tabular}{|c|c|c|c|c|c|c|c|c|c|c|c|c|}
\hline 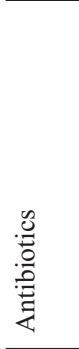 & 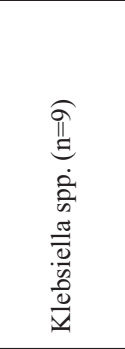 & 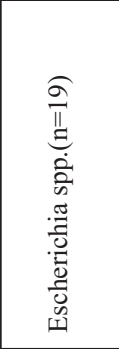 & 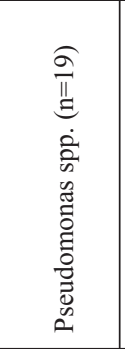 & 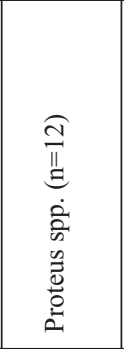 & 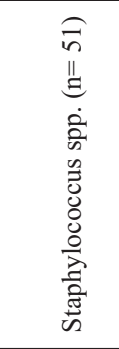 & 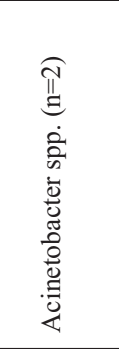 & 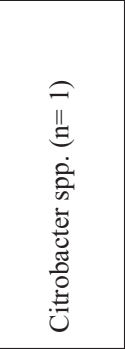 & 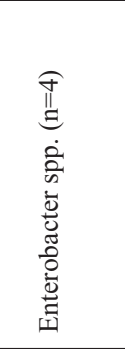 & 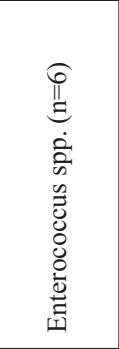 & 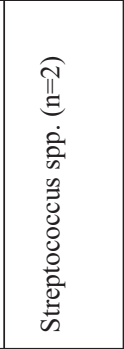 & 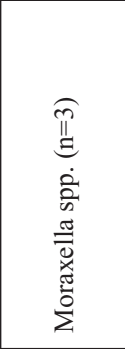 & 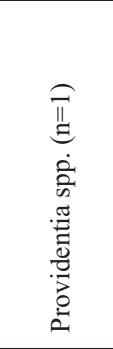 \\
\hline NET & $\mathrm{R}(100 \%)$ & $\mathrm{S}(90 \%)$ & $\mathrm{S}(70 \%)$ & $\mathrm{S}(70 \%)$ & $\mathrm{S}(90 \%)$ & $\mathrm{S}(100 \%)$ & $\mathrm{R}(100 \%)$ & $\mathrm{R}(75 \%)$ & $\mathrm{S}(100 \%)$ & $\mathrm{S}(100 \%)$ & $\mathrm{S}(100 \%)$ & $\mathrm{R}(100 \%)$ \\
\hline $\mathrm{CN}$ & $\mathrm{R}(100 \%)$ & $\mathrm{S}(90 \%)$ & $\mathrm{S}(80 \%)$ & $\mathrm{S}(80 \%)$ & $\mathrm{S}(80 \%)$ & $\mathrm{R}(100 \%)$ & $\mathrm{R}(100 \%)$ & $\mathrm{R}(100 \%)$ & $\mathrm{S}(80 \%)$ & $\mathrm{S}(100 \%)$ & $\mathrm{S}(100 \%)$ & $\mathrm{R}(100 \%)$ \\
\hline $\mathrm{AK}$ & $\mathrm{R}(70 \%)$ & $\mathrm{S}(70 \%)$ & $\mathrm{S}(90 \%)$ & $\mathrm{S}(100 \%)$ & $\mathrm{S}(70 \%)$ & $\mathrm{R}(100 \%)$ & $\mathrm{R}(100 \%)$ & $R(75 \%)$ & $\mathrm{R}(100 \%)$ & $\mathrm{R}(50 \%)$ & $\mathrm{S}(100 \%)$ & $\mathrm{R}(100 \%)$ \\
\hline IMP & $\mathrm{R}(70 \%)$ & $\mathrm{S}(80 \%)$ & $\mathrm{S}(90 \%)$ & $\mathrm{S}(100 \%)$ & - & $\mathrm{R}(100 \%)$ & $\mathrm{R}(100 \%)$ & $\mathrm{S}(100 \%)$ & - & - & $\mathrm{S}(100 \%)$ & $\mathrm{S}(100 \%)$ \\
\hline $\mathrm{TZP}$ & $\mathrm{R}(100 \%)$ & $\mathrm{S}(100 \%)$ & $\mathrm{R}(100 \%)$ & $\mathrm{S}(100 \%)$ & - & $\mathrm{R}(100 \%)$ & $\mathrm{R}(100 \%)$ & $\mathrm{S}(100 \%)$ & - & - & & $\mathrm{S}(100 \%)$ \\
\hline CFM & $\mathrm{R}(70 \%)$ & $\mathrm{R}(100 \%)$ & $\mathrm{R}(100 \%)$ & $\mathrm{R}(80 \%)$ & - & $\mathrm{R}(100 \%)$ & $\mathrm{R}(100 \%)$ & $\mathrm{S}(75 \%)$ & - & - & $\mathrm{R}(100 \%)$ & $\mathrm{R}(100 \%)$ \\
\hline $\mathrm{AMC}$ & $\mathrm{R}(90 \%)$ & $\mathrm{R}(90 \%)$ & $\mathrm{R}(90 \%)$ & $\mathrm{R}(70 \%)$ & $\mathrm{R}(60 \%)$ & $\mathrm{R}(100 \%)$ & $\mathrm{R}(100 \%)$ & $\mathrm{R}(75 \%)$ & - & - & $\mathrm{R}(100 \%)$ & $\mathrm{R}(100 \%)$ \\
\hline CRO & $\mathrm{R}(100 \%)$ & $\mathrm{R}(100 \%)$ & $\mathrm{R}(80 \%)$ & $\mathrm{R}(70 \%)$ & - & $\mathrm{R}(100 \%)$ & $\mathrm{R}(100 \%)$ & $\mathrm{S}(100 \%)$ & - & - & $\mathrm{R}(100 \%)$ & $\mathrm{R}(100 \%)$ \\
\hline CAZ & $\mathrm{R}(100 \%)$ & $\mathrm{R}(80 \%)$ & $\mathrm{R}(90 \%)$ & $\mathrm{R}(60 \%)$ & - & $\mathrm{R}(100 \%)$ & $\mathrm{R}(100 \%)$ & $\mathrm{R}(100 \%)$ & - & - & $\mathrm{R}(100 \%)$ & $\mathrm{R}(100 \%)$ \\
\hline CTx & $\mathrm{R}(90 \%)$ & $R(100 \%)$ & $\mathrm{R}(100 \%)$ & $\mathrm{R}(50 \%)$ & - & $\mathrm{R}(100 \%)$ & $\mathrm{R}(100 \%)$ & $\mathrm{S}(100 \%)$ & - & - & $\mathrm{R}(100 \%)$ & $\mathrm{R}(100 \%)$ \\
\hline FEP & $\mathrm{R}(60 \%)$ & $\mathrm{R}(70 \%)$ & $\mathrm{R}(70 \%)$ & $\mathrm{R}(50 \%)$ & - & $\mathrm{R}(100 \%)$ & $\mathrm{R}(100 \%)$ & $\mathrm{S}(100 \%)$ & - & - & $\mathrm{R}(100 \%)$ & $\mathrm{R}(100 \%)$ \\
\hline CT & $\mathrm{S}(100 \%)$ & $\mathrm{S}(100 \%)$ & $\mathrm{S}(100 \%)$ & $\mathrm{R}(100 \%)$ & $\mathrm{R}(70 \%)$ & $\mathrm{S}(100 \%)$ & $\mathrm{S}(100 \%)$ & $\mathrm{S}(100 \%)$ & - & - & $\mathrm{R}(100 \%)$ & $\mathrm{R}(100 \%)$ \\
\hline SXT & $\mathrm{R}(100 \%)$ & $\mathrm{R}(80 \%)$ & $\mathrm{S}(80 \%)$ & $\mathrm{R}(100 \%)$ & $\mathrm{S}(80 \%)$ & $\mathrm{R}(100 \%)$ & $\mathrm{R}(100 \%)$ & $\mathrm{R}(100 \%)$ & $\mathrm{R}(80 \%)$ & $\mathrm{R}(100 \%)$ & $S(100 \%)$ & $\mathrm{R}(100 \%)$ \\
\hline CIP & $\mathrm{R}(90 \%)$ & $\mathrm{R}(90 \%)$ & $\mathrm{S}(80 \%)$ & $\mathrm{R}(70 \%)$ & $\mathrm{S}(70 \%)$ & $\mathrm{R}(100 \%)$ & $\mathrm{R}(100 \%)$ & $\mathrm{R}(100 \%)$ & $\mathrm{S}(100 \%)$ & $\mathrm{S}(100 \%)$ & $S(100 \%)$ & $\mathrm{R}(100 \%)$ \\
\hline OX & - & - & $\mathrm{S}(100 \%)$ & - & $\mathrm{R}(80 \%)$ & - & - & - & - & - & - & - \\
\hline FOX & - & - & $\mathrm{S}(90 \%)$ & - & $\mathrm{R}(80 \%)$ & - & - & - & - & - & - & - \\
\hline $\mathrm{CL}$ & - & - & $\mathrm{R}(100 \%)$ & $\mathrm{R}(100 \%)$ & $\mathrm{R}(70 \%)$ & - & - & - & - & - & - & - \\
\hline CXM & - & - & $\mathrm{R}(100 \%)$ & - & $\mathrm{R}(80 \%)$ & - & & - & - & - & - & - \\
\hline FUS & - & - & $\mathrm{R}(100 \%)$ & - & $\mathrm{S}(60 \%)$ & - & - & - & - & - & - & - \\
\hline $\mathrm{RD}$ & - & - & & - & $\mathrm{S}(80 \%)$ & - & - & - & - & - & - & - \\
\hline VA & - & - & $\mathrm{S}(100 \%)$ & - & $\mathrm{S}(90 \%)$ & - & - & - & $\mathrm{S}(100 \%)$ & $\mathrm{S}(100 \%)$ & - & - \\
\hline DA & - & - & $\mathrm{R}(100 \%)$ & - & $\mathrm{S}(70 \%)$ & - & - & - & - & - & - & - \\
\hline RA & - & - & $\mathrm{R}(100 \%)$ & - & $\mathrm{S}(90 \%)$ & - & - & - & - & - & - & - \\
\hline TOB & - & - & - & - & - & - & - & $\mathrm{R}(100 \%)$ & - & - & - & $\mathrm{R}(100 \%)$ \\
\hline ATM & - & - & - & - & - & - & - & $\mathrm{R}(75 \%)$ & - & - & - & $\mathrm{R}(100 \%)$ \\
\hline TGC & - & - & - & - & - & - & - & $\mathrm{S}(100 \%)$ & - & - & - & $\mathrm{S}(100 \%)$ \\
\hline CAR & - & - & - & - & - & - & - & $\mathrm{R}(100 \%)$ & - & - & - & $\mathrm{R}(100 \%)$ \\
\hline FD & - & - & - & - & $\mathrm{R}(100 \%)$ & - & - & - & - & - & - & - \\
\hline
\end{tabular}

NET $=$ Netilmicin, $\mathrm{SxT}=$ Trimethoprim-Sulphamethoxazole, $\mathrm{FUS}=$ Fusidic acid, $\mathrm{CIP}=$ Ciprofloxacin, $\mathrm{Ox}=$ Oxacillin, $\mathrm{VA}=\mathrm{Vancomycin}, \mathrm{TOB}=\mathrm{Tobramycin}, \mathrm{CAR}=$ Carbenicillin, $\mathrm{IMP}=$ Imipenem, $\mathrm{CFM}=$ Cefalonium, $\mathrm{AMC}=$ Amoxicillin, $\mathrm{ATM}=$ Azithromycin $, \mathrm{CRO}=\mathrm{Ceftriaxone}, \mathrm{TGC}=\mathrm{Tigecycline}, \mathrm{CTx}=\mathrm{Cefotaxime}, \mathrm{FOX}=$ Cefoxitin, $\mathrm{CXM}=$ Cefuroxime, $\mathrm{TZP}=$ Piperacillin-Tazobactam, $\mathrm{CAZ}=$ Ceftazidime, $\mathrm{FEP}=$ Cefepime, $\mathrm{FD}=\mathrm{Fusidic}$ acid, $\mathrm{DA}=\mathrm{Clindamycin}, \mathrm{CL}=\mathrm{Ch} l o r e m p h e n i c o l$, $\mathrm{AK}=$ Amikacin, $\mathrm{CT}=$ Colistin, $\mathrm{CN}=$ Gentamicin, $\mathrm{RA}=$ Rifampin, $\mathrm{RD}=$ Radicicol 
From the result table, it is clear to understand that all of the isolates have already become resistant to many antibiotics which are used very commonly in our country. Many drugs have become $100 \%$ resistant according to the isolates. For example, the isolates showed $100 \%$ resistance towards CAR, ATM, TOB, CXM, FD, CL, CAZ, AMC. NET, CN, IMP have been shown to be the most effective drugs to which maximum isolates were susceptible. All the isolates were susceptible to VA (Pseudomonas spp., S. aureus, S. epidermidis, Enterococcus spp., S. pyogenes, S. agalactiae) which were subjected to it, Enterococcus spp., Providentia spp., Staphylococcus spp. towards RD. $75 \%$ to $90 \%$ resistance was shown to other antibiotics.

\section{Discussion}

To treat the bacterial infection antibiotic administration is the most effective way. But the effectiveness has been compromised with the drug resistance traits of the pathogenic isolates which render the infections difficult to treat. As a result mortality rate due to the infections are rising. In the present study, 100 patients (both male and female) having blood borne infections were selected to determine the causative agent of the bacteremia as well as the degree of antibiotic drug resistance traits. Shockingly it was observed that the bacterial isolates found from the blood samples are highly resistant towards the antibiotics which are popularly used by the physicians to treat bacteremic patients. For example, the most predominant pathogen Staphylococcus aureus was already resistant to seven popularly used antibiotics (AMC, CT, OX, FOX, CL, CXM, FD). In contrast, Providentia spp. was susceptible only to two antibiotics (IMP, TZP). One of the most common pathogenic agents Klebsiella spp. was found to be susceptible only to the antibiotic CT. This kind of finding represents an alarming condition of Bangladesh where treating bacteremic patients is getting difficult and if not controlled the treatment procedure will become much more challenging.

From Table 04 it is clear that the bacteria found in blood, showed resistance towards different antibiotics From this table we can state that the drug resistance pattern is growing very fast and the time is not so far when all the remaining susceptible antibiotics will also become ineffective.

The resistance mechanisms are different for different organisms. For example, Pseudomonas aeruginosa can survive by multidrug efflux mechanism controlled by the transcription regulator encouraging the drug resistant gene expression ${ }^{53-56}$. Salmonella enterica has been found to be resistant against â lactam antibiotics by producing â lactamases ${ }^{57-59}$. Many pathogenic isolates can produce aminoglycoside modifying enzymes which add the features of aminoglycoside resistance ${ }^{32}$. Aminoglycoside 6- $N$ acetyltransferase type $\mathrm{Ib}$ is now a clinically important enzyme which has been found in many Gram positive bacteria which confer them resistance to aminoglycosides ${ }^{60}$. Mutations in ribosomal protein S 5in Neisseria gonorrhoeae decrease susceptibility to spectinomycin, cefixime and ceftriaxone ${ }^{61}$. Methicillin resistant Staphylococcus aureus is also very common in the world 62 .

Drug resistance genes can be transferred to other susceptible microorganisms with the help of plasmids and virus. Genetic recombination, insertion of new resistant genes are very common in the case of transferring new drug resistant genes. Mutations in

Table 3. Distribution of pathogenic bacteria causing bacteremia in Dhaka city from 2005 to $2016^{51}$.

\begin{tabular}{|c|c|c|c|}
\hline Etiological agents & $\begin{array}{l}\text { Bacteremia during } \\
\text { the time period of } \\
2005 \text { to } 2014\end{array}$ & Bacteremia in 2016 & Changes \\
\hline $\begin{array}{l}\text { Most } \\
\text { predominating } \\
\text { pathogenic } \\
\text { bacteria }\end{array}$ & Salmonella Typhi & $\begin{array}{l}\text { Staphylococcus } \\
\text { aureus }\end{array}$ & $\begin{array}{l}\text { Blood borne infection by Salmonella spp. } \\
\text { drastically decreased and previously common } \\
\text { etiologic agent Staphylococcus spp. } \\
\text { (S. aureus) has become the most predominant } \\
\text { pathogenic bacteria. }\end{array}$ \\
\hline $\begin{array}{l}\text { Other frequently } \\
\text { isolated bacteria }\end{array}$ & $\begin{array}{l}\text { Staphylococcus spp., } \\
\text { Pseudomonas spp., } \\
\text { Acinetobacter spp., } \\
\text { Salmonella paratyphi } \\
\text { A,B, Klebsiella spp, } \\
\text { E. coli, Enterobacter } \\
\text { spp., Serratia spp, } \\
\text { Klebsiella spp. }\end{array}$ & $\begin{array}{l}\text { Pseudomonas, E. } \\
\text { coli, Proteus } \\
\text { vulgaris, Klebsiella } \\
\text { spp. }\end{array}$ & $\begin{array}{l}\text { Some commonly found bacteria are steadily } \\
\text { causing bacteremia (Pseudomonas, E. coli, } \\
\text { Klebsiella spp.). New agent has been emerged } \\
\text { such as Proteus vulgaris. }\end{array}$ \\
\hline $\begin{array}{l}\text { Occasionally } \\
\text { found pathogenic } \\
\text { bacteria }\end{array}$ & Streptococcus spp. & $\begin{array}{l}\text { Enterococcus spp., } \\
\text { Enterobacter spp., } \\
\text { Streptococcus spp., } \\
\text { Acinetobacter spp., } \\
\text { Citrobacter spp., } \\
\text { Providentia spp. }\end{array}$ & $\begin{array}{l}\text { New groups of bacteria emerged (Citrobacter } \\
\text { spp., Providentia spp.). Previous frequently } \\
\text { encountered bacteria (Enterobacter spp., } \\
\text { Acinetobacter spp.) decreased and become } \\
\text { occasional pathogenic agents. }\end{array}$ \\
\hline
\end{tabular}


Table 4. Changes in the drug resistance pattern from the past to the recent year-2016 in perspective of Bangladesh $51,52$.

\begin{tabular}{|c|c|c|}
\hline Pathogenic bacteria & $\begin{array}{l}\text { Increasing trend of resistance to } \\
\text { antibiotics before } 2016\end{array}$ & $\begin{array}{l}\text { Resistance to antibiotics in the } \\
\text { year of } 2016\end{array}$ \\
\hline Escherichia coli & $\begin{array}{l}\text { Ampicillin, Gentamicin, Ciprofloxacin, } \\
\text { Ceftriaxone, Netilmicin, Amikacin, } \\
\text { Imipenem, Ceftazidime, Cefixime. }\end{array}$ & $\begin{array}{l}\text { Trimethoprim-Sulphamethoxazole, } \\
\text { Ciprofloxacin, Cefalonium, } \\
\text { Amoxicillin, Ceftriaxone, Cefotaxime, } \\
\text { Ceftazidime, Cefepime. }\end{array}$ \\
\hline Staphylococcus aureus & $\begin{array}{l}\text { Trimethoprim-Sulphamethoxazole, } \\
\text { Ciprofloxacin, Ceftriaxone, } \\
\text { Chloremphenicol, Gentamicin, } \\
\text { Erythromycin }\end{array}$ & $\begin{array}{l}\text { Oxacillin, Amoxicillin, Cefoxitin, } \\
\text { Cefuroxime, Fusidic acid, } \\
\text { Chloremphenicol, Colistin. }\end{array}$ \\
\hline Streptococcus spp. & Erythromycin, Azithromycin & $\begin{array}{l}\text { Trimethoprim-Sulphamethoxazole, } \\
\text { Netilmicin, Amikacin }\end{array}$ \\
\hline Enterococcus spp. & $\begin{array}{l}\text { Fusidic acid, Amikacin, Gentamicin, } \\
\text { Netilmicin, Ciprofloxacin, } \\
\text { Chloramphenicol, cotrimoxazole. }\end{array}$ & $\begin{array}{l}\text { Amikcin, } \\
\text { Trimethoprim-Sulphamethoxazole. }\end{array}$ \\
\hline Pseudomonas spp. & $\begin{array}{l}\text { Aztreonam, , cefotaxime, ceftriaxone, } \\
\text { chloramphenicol, , colistin, gentamicin, } \\
\text { imipenem, netilmicin }\end{array}$ & $\begin{array}{l}\text { Fusidic acid, Cefalonium, Amoxicillin, } \\
\text { Ceftriaxone, Cefotaxime, Cefuroxime, } \\
\text { Piperacillin-Tazobactam, Ceftazidime, } \\
\text { Cefepime, Clindamycin, } \\
\text { Chloremphenicol, Rifampin. }\end{array}$ \\
\hline Acinetobacter spp. & $\begin{array}{l}\text { Cefotaxime, Ceftriaxone, Ciprofloxacin, } \\
\text { Gentamicin, } \\
\text { Netilmicin, Piperacillin and Tazobactam } \\
\text { combination, Amikacin, Ceftazidime }\end{array}$ & $\begin{array}{l}\text { Trimethoprim-Sulphamethoxazole } \\
\text { Ciprofloxacin, Imipenem, Cefalonium, } \\
\text { Amoxicillin, Ceftriaxone, Cefotaxime, } \\
\text { Piperacillin-Tazobactam, Ceftazidime, } \\
\text { Cefepime, Amikacin, Gentamicin, }\end{array}$ \\
\hline
\end{tabular}

the genetic material is also another mechanism. Such conditions have worsened the course of treatment due to lack of effective antibiotics. New drugs are needed to be produced for such conditions.

Resistant bacteria can transmit to other healthcare workers accidentally by sharps and they (healthcare workers) can transfer these bacteria to other patients if proper protective clothing and apparatus are not used. In medicals the unsterilized patient samples and garbage containing the contaminants may also allow the drug resistant pathogens to spread in the environment. So medical disposals should be under strict supervision of law and the medical authority. People infected with the drug resistant bacteria can be treated with only a few antibiotics This condition is driving the scientists to discover new antibiotics to treat drug resistant isolates.

\section{Conclusion}

The present study reflects a very alarming scenario in the medical science where almost all kinds of pathogenic bacteria responsible for causing bacteremia have become resistant to a wide range of antibiotics. Only a few antibiotics are till now effective. But there is a chance of these antibiotics to become resistant in the future. So necessary steps should be followed by the healthcare professionals and patients together in taking the medications and awareness about the transmissions.
The complications of drug resistance has become a major threat in Bangladesh and it is necessary to get the attention of the scientific community to understand the upcoming devastating condition in the medical era. About 100 bacteremic patients who were subjected to drug resistance test before treatment, alarmingly showed that all pathogenic bacteria responsible for causing bacteria were resistant towards maximum types of antibiotics which are generally prescribed in our country. A large number of patients are in a life threatening condition because the infection is not recovering by the conventional antibiotic therapy. Moreover, the resistance genes can render other susceptible bacteria into resistant ones through plasmids and other mobile elements. So, it is needed to check this problem by proper dosage, patient awareness and investigation for newer therapeutics.

\section{Acknowledgement}

We are thankful to the authors whose research articles and reports have been used to cite in this study.

\section{References}

1. Seifert H. 2009. The Clinical Importance of Microbiological Findings in the Diagnosis and Management of Bloodstream Infections. Clinical Infectious Diseases, 48:238-45.

2. Martin GS, Mannino DM, Eaton S and Moss M. 2003. The epidemiology of sepsis in the United States from 1979 through 2000. N. Engl. J. Med., 348:1546-1554. 
3. Mootsikapun P. 2007. Bacteremia in adult patients with acquired i $\mathrm{m} \mathrm{m}$ u n o d e fi c i e n c y s y n d r o m e in the northeast of Thailand. Int $J l$ Infect Dis., 11: 226-231

4. Naber CK. 2009. Staphylococcus aureus Bacteremia: Epidemiology, Pathophysiology, and Management Strategies. Clinical Infectious Diseases, 48: 231-7.

5. Wisplinghoff H, Bischoff T, Tallent SM, Seifert H, Wenzel RP and Edmond MB. 2004. Nosocomial bloodstream infections in US hospitals: analysis of 24,179 cases from a prospective nationwide surveillance study. Clin Infect Dis., 39: 309-17.

6. Hossain B, Weber MW, Hamer DH, Hibberd LP, Ahmed ASMNW, Marzan M, Islam M, NicConnor EN, Islam SM, Zaidi AK, Baqui AH, Bhutta ZA, Qureshi MS, Rafiullah I, McGee L and Saha SK. 2016. Classifiation of Blood Culture Isolates Into Contaminants and Pathogens on the Basis of Clinical and Laboratory Data. Pediatr Infect Dis J., 35: S52-S54

7. Shorr AF and Lodise T. 2006. Burden of methicillin-resistant Staphylococcus aureus on healthcare cost and resource utilization. ISMR Update., 1: 4-11.

8. EARSS management team. 2006. European Antimicrobial Resistance Surveillance System annual report 2006. Bilthoven, The Netherlands: National Institute for Public Health and the Environment, 2007.

9. Troidle L, Eisen T, Pacelli L and Finkelstein F. 2007. Complications associated with the development of bacteremia with Staphylococcus aureus. Hemodial Int., 11: 72-5.

10. Fowler VG Jr, Miro JM, Hoen B, Cabell CH, Abrutyn E, Rubinstein E et al. 2005. Staphylococcus aureus endocarditis: a consequence of medical progress. JAMA., 293: 3012-21.

11. Tacconelli E, Venkataraman L, De Girolami PC DA DAgata EM. 2004. Methicillin-resistant Staphylococcus aureus bacteraemia diagnosed at hospital admission: distinguishing between community-acquired versus healthcare-associated strains. J Antimicrob Chemother, 53: 474-9.

12. Carnicer-Pont D, Bailey KA, Mason BW, Walker AM, Evans MR and Salmon RL. 2006. Risk factors for hospital-acquired methicillin-resistant Staphylococcus aureus bacteraemia: a case-control study. Epidemiol Infect., 134: 1167-73.

13. Mitchell DH and Howden BP. 2005. Diagnosis and management of Staphylococcus aureus bacteraemia. Intern Med J., 35(2): 17-24.

14. Biedenbach DJ, Moet GJ and Jones RN. 2004. Occurrence and antimicrobial resistance pattern comparisons among bloodstream infection isolates from the SENTRY Antimicrobial Surveillance Program (19972002). Diagn. Microbiol. Infect. Dis., 50: 59-69.

15. Diekema DJ. 2003. Epidemiology and outcome of nosocomial and community-onset bloodstream infection. J. Clin. Microbiol., 41: 36553660 .

16. Javaloyas M, Garcia-Somoza D and Gudiol F. 2002. Epidemiology and prognosis of bacteremia: a 10-y study in a community hospital. Scand. J. Infect. Dis., 34: 436-441.

17. Laupland KB and Church DL. 2007. Burden of community-onset bloodstream infection: a population-based assessment. Epidemiol. Infect., 135: $1037-1042$

18. Lyytikainen O, Lumio J, Sarkkinen H, Kolho E, Kostiala A and Ruutuet P. 2002. Nosocomial bloodstream infections in Finnish hospitals during 1999-2000. Clin. Infect. Dis., 35: 14-e19.

19. Uslan DZ, Crane SJ, Steckelberg JM, Cockerill FR, Sauver JL, Wilson WR, et al. 2007. Age- and sex-associated trends in bloodstream infection: a population-based study in Olmsted County, Minnesota. Arch Intern Med., 167: 834-839.

20. Lefort A, Panhard X, Clermont O, Woerther PL, Branger C, Mentre' F, et al. 2011. Host Factors and Portal of Entry Outweigh Bacterial Determinants To Predict the Severity of Escherichia coli Bacteremia. Journal of Clinical Microbiology., 777-783
21. Hendriksen RS, Hyytia-Trees E, Pulsrikarn C, Pornruangwong S, Chaichana S, Svendsen CA, Ahmed R and Mikoleit M. 2012. Characterization of Salmonella enterica serovar Enteritidis isolates recovered from blood and stool specimens in Thailand. $B M C$ Microbiology., 12: 92.

22. Gordon MA, Banda HT, Gondwe M, Gordon SB, Boeree MJ, Walsh AL, et al. 2002. Non-typhoidal salmonella bacteraemia among HIVinfected Malawian adults: high mortality and frequent recrudescence. AIDS., 16: $1633-41$.

23. Anglaret X, Messou E, Ouassa T, Toure S, Dakoury-Dogbo N, Combe P, et al. 2003. Pattern of bacterial diseases in a cohort of HIV-1 infected adults receiving co-trimoxazole prophylaxis in Abidjan, Cote d'Ivoire. AIDS., 17: 575-84.

24. Benson AC, Kaplan JE, Masur H, Pau A and Holms KK. 2004. Treating opportunistic infections among HIV-exposed and infected children: recommendations from $\mathrm{CDC}$, the National Institutes of Health, and the Infectious Diseases Society of America. MMWR Recomm Rep., 53 (RR15): $1-112$.

25. Tumbarello M, Tacconelli E, Donati KG, Citton R, Leone F, Spanu T et al. 2000. HIV-associated bacteremia: how it has changed in the highly active antiretroviral therapy (HAART) era. J Acquir Immune Defic Syndr., 23: $145-51$

26. Meynard JL, Guiguet M, Fonquernie L, Lefebvre V, Lalande V, Honore I, et al. 2003. Impact of highly active antiretroviral therapy on the occurrence of bacteraemia in HIV-infected patients and their epidemiologic characteristics. H I V M e d., 4: 127-32.

27. Carney K, Dhalla S, Aytaman A, Tenner CT and Francois FA. 2013. Association of tattooing and hepatitis $\mathrm{C}$ virus infection: a multicenter casecontrol study. Hepatol J., 57(6): 2117-23.

28. Bacusca AI, Coman AE, Felea D, Petrovanu R and Ioan B. 2014. Epidemiology of $\mathrm{B} / \mathrm{C}$ virus infection hepatitis in the Northern Moldavian correctional facilities risk factors. Rev Med Chir Soc Med Nat Iasi., 118(2): 463-70.

29. Nokhodian Z, Meshkati M, Adibi P, Ataei B, Kassaian N, Yaran M, et al. 2012. Hepatitis $C$ among Intravenous Drug Users in Isfahan, Iran: a Study of Seroprevalence and Risk Factors. Int J Prev Med., 3(1): 131-8. [PubMed: 22826755]

30. Matos SB, Jesus AL, Pedroza KC, Sodre HR, Ferreira TL and Lima FW. 2013. Prevalence of serological markers and risk factors for bloodborne pathogens in Salvador, Bahia state, Brazil. Epidemiol Infect., 141(1): 181-7.

31. Mahfoud Z, Kassak K, Kreidieh K, Shamra S and Ramia S. Prevalence of antibodies to human immunodefiiency virus (HIV), hepatitis B and hepatitis $\mathrm{C}$ and risk factors in prisoners in Lebanon. J Infect Dev Ctries., 4(3): 144-9.

32. Shi MD, Lee SY and Lee YB. 2007. Increased risk of viral hepatitis in Taiwanese male conscriptees with tattoos. Mil Med., 172(5): 539-40.

33. Viana S, Parana R, Moreira RC, Compri AP and Macedo V. 2005. High prevalence of hepatitis B virus and hepatitis D virus in the western Brazilian Amazon. Am J Trop Med Hyg., 73(4): 808-14.

34. Nishioka Sde A, Gyorkos TW, Joseph L, Collet JP and Maclean JD. 2002. Tattooing and risk for transfusion-transmitted diseases: the role of the type, number and design of the tattoos, and the conditions in which they were performed. Epidemiol Infect., 128(1): 63-71.

35. Tohme RA and Holmberg SD. Transmission of hepatitis $\mathrm{C}$ virus infection through tattooing and piercing: a critical review. Clin Infect Dis., 54(8): $1167-78$.

36. Urbanus AT, van den Hoek A, Boonstra A, van Houdt R, de Bruijn LJ, Heijman T, et al. 2011. People with multiple tattoos and/or piercings are not at increased risk for HBV or HCV in The Netherlands. PLoS One, 6(9): 24736.

37. Pena-Orellana M, Hernandez-Viver A, Caraballo-Correa G and AlbizuGarcia CE. 2011. Prevalence of HCV risk behaviors among prison inmates: tattooing and injection drug use. $J$ Health Care Poor Underserved., 22(3): 962-82. 
38. Haddadin AS, Fappiano SA and Lipsett PA. 2002. Methicillin resistant Staphylococcus aureus (MRSA) in the intensive care unit. Postgrad Med J., 78: 385-92

39. Weidenmaier C, Kokai-Kun JF, Kristian SA, Chanturiya T, Kalbacher H, Gross M. 2004. Role of teichoic acids in Staphylococcus aureus nasal colonization, a major risk factor in nosocomial infections. Nat Med., 10: 243-5.

40. Amagai M, Matsuyoshi N, Wang ZH, Andl C and Stanley JR. 2000. Toxin in bullous impetigo and staphylococcal scalded-skin syndrome targets desmoglein 1. Nat Med., 6: 1275-7.

41. Otto M. 2004. Quorum-sensing control in staphylococci-a target for antimicrobial drug therapy. FEMS Microbiol Lett., 241: 135-41

42. Haas PJ, de Haas CJ, Kleibeuker W, Poppelier MJ, van Kessel KP, Kruijtzer JA, et al. 2004. N-terminal residues of the chemotaxis inhibitory protein of Staphylococcus aureus are essential for blocking formylated peptide receptor but not C5a receptor. J Immunol., 173: 5704-11

43. McCormick JK, Yarwood JM and Schlievert PM. 2001. Toxic shock syndrome and bacterial superantigens: an update. Annu Rev Microbiol., 55: $77-104$

44. Kempf VA, Trebesius K and Autenrieth IB. 2000. Fluorescent in situ hybridization allows rapid identification of microorganisms in blood cultures. J Clin Microbiol., 38: 830-8.

45. Wellinghausen N, Wirths B, Essig A and Wassill L. 2004. Evaluation of the Hyplex BloodScreen multiplex PCR-enzyme-linked immunosorbent assay system for direct identification of gram-positive cocci and gramnegative bacilli from positive blood cultures. J Clin Microbiol., 42: $3147-$ 52 .

46. Shrestha NK, Tuohy MJ, Hall GS, Isada CM and Procop GW. 2002. Rapid identification of Staphylococcus aureus and the mecA gene from BacT/ ALERT blood culture bottles by using the LightCycler system. $J$ Clin Microbiol., 40: 2659-61.

47. National Nosocomial Infections Surveillance System. 2004. National Nosocomial Infections Surveillance (NNIS) System Report, data summary from January 1992 through June 2004, issued October 2004. Am. J. Infect. Control., 32: 470-485.

48. Hall KK and Lyman JA. 2006. Updated Review of Blood Culture Contamination. Clin Microbiol Rev., 19(4): 788-802.

49. Hall G, Heimdahl A and Nord CE. 1999. Bacteremia after oral surgery and antibiotic prophylaxis for endocarditis. Clin Infect Dis., 29:1-10.

50. Petti CA, Woods CW and Reller LB. Streptococcus pneumoniae antigen test using positive blood culture bottles as an alternative method to diagnose pneumococcal bacteremia. J Clin Microbiol., 43(5): 2510-2.
51. Ahmed D, Ausrafuggaman MN, Abdullah BS, Farhana H, Nasrin A, et al. 2017. Bacterial etiology of bloodstream infections and antimicrobial resistance in Dhaka, Bangladesh, 2005-2014. Antimicrobial resistance and infection control., 6 : 2 .

52. Arif A, Kaniz F, Lovely B, Mohammad OF, Fatema A, et al. 2016. Prevalence and Antimicrobial Resistance Pattern of Blood Isolates in Patients of Septicemia in ICU: Single Centre Observation. Bangladesh Crit Care J., 4(2): 100-104.

53. Morita Y, Tomida J and Kawamura Y. 2012. MexXY multidrug efflux system of Pseudomonas aeruginosa. Front. Microbiol., 3: 408.

54. Usui M, Nagai H, Hiki M, Tamura Y and Asai T. 2 013. Effect of antimicrobial exposure on AcrAB Expression in Salmonella enterica subspecies enteric serovar choleraesuis. Front. Microbiol., 4: 53.

55. Deng Z, Shan Y, Pan Q, Gao X and Yan A. 2013. Anaerobic expression of the gadE-mdtEF multidrug efflux operon is primarily regulated by the twocomponent system ArcBA through antagonizing the H-NS mediated repression. Front. Microbio. 4: 194.

56. Baucheron S, Monchaux I, Le HS, Weill FX and Cloeckaert A. 2014. Lack of efflux mediated quinolone resistance in Salmonella enterica serovars Typhi and Paratyphi A. Front. Microbiol., 5: 12.

57. Chuma T, Miyasako D, Dahshan H, Takayama T, Nakamoto Y, Shahada F. et al. 2013. Chronological change of resistance to beta-Lactams in Salmonella enterica serovar infantis isolated from broilers in Japan. Front. Microbiol., 4: 113.

58. Watkins RR, Papp-Wallace KM, Drawz SM and Bonomo RA. 2013. Novel beta-lactamase inhibitors: a therapeutic hope against the scourge of multidrug resistance. Front. Microbiol., 4: 392.

59. Zeng $X$ and Lin J. 2013. Beta-lactamase induction and cell wall metabolism in Gram-negative bacteria. Front. Microbiol., 4: 128.

60. Ramirez MS, Nikolaidis N and Tolmasky ME. 2013. Rise and dissemination of aminoglycoside resistance: the aac(6 )-Ib paradigm. Front. Microbiol., 4: 121

61. Zaheer R, Cook SR, Klima CL, Stanford K, Alexander T, Topp E, et al. 2013. Effect of subtherapeutic vs. therapeutic administration of macrolides on antimicrobial resistance in Mannheimia haemolytica and enterococci isolated from beef cattle. Front. Microbiol., 4: 133.

62. Roberts MC, Soge OO and No D. 2014. Comparison of multi-drug resistant environmental methicillin-resistant Staphylococcus aureus isolated from recreational beaches and high touch surfaces in built environments. Front. Microbiol., 4: 74. 